\section{Repercusión de la educación virtual en estudiantes del área de Ciencias de la Salud tras la pandemia por SARS-CoV-2}

\author{
Impact of virtual education on students in \\ the health sciences area after the SARS- \\ CoV-2 pandemic
}

\section{Resumen}

La enfermedad por coronavirus 2019 (COVID-19) es una infección respiratoria aguda altamente transmisible, la cual ha sido declarada pandemia por la Organización Mundial de la Salud. En concordancia con las políticas gubernamentales para la prevención sanitaria y aislamiento social, se aprobó la virtualidad de todo el sistema de educación, incluyendo carreras con un fuerte componente práctico como las de Ciencias de la Salud; lo cual conllevo a la adaptación de planes y programas de estudio, así como a la inmersión de utilización de plataformas virtuales, generando un descubrimiento de nuevas formas de aprendizaje tanto en el área teórica y práctica, sin embargo al mismo tiempo se generaron ciertas repercusiones en destrezas y habilidades de tipo prácticas; abriendo además, una brecha entre la desigualdad en el acceso de las tecnologías.

Palabras clave: Educación virtual; Pandemia; COVID-19; Estudiantes; Ciencias de la salud (fuente: DeCS BIREME).

\begin{abstract}
Coronavirus disease 2019 (COVID-19) is a highly communicable acute respiratory infection, which has been declared a pandemic by the World Health Organization. In accordance with government policies for health prevention and social isolation, the virtuality of the entire education system was approved, including careers with a strong practical component such as health sciences; which led to the adaptation of study plans and programs, as well as the immersion of the use of virtual platforms, generating a discovery of new forms of learning both in the theoretical and practical areas, however, at the same time, certain repercussions were generated in practical and psychomotor skills and abilities; also opening a gap between inequality in access to technologies.
\end{abstract}

Keywords: Virtual education; Pandemic; COVID-19; Students; Health sciences (source: MeSH NLM).

\section{Nota Científica}

Graciela Zambrano-Galván ${ }^{1, a}$, Martha Angélica Quintanar-Escorza ${ }^{1, b}$, Laura Ernestina Barragán Ledesma ${ }^{1, c}$

${ }^{1}$ Universidad Juárez del Estado de Durango, Facultad de Medicina y Nutrición, Durango, Dgo, México.

a Doctor en Ciencias Biomédicas.

b Doctor en Ciencias en Bioquímica.

' Doctor en Ciencias de la Salud Pública.

\section{Correspondencia:}

Graciela Zambrano-Galván: gzambrano@ujed.mx Av. Universidad y Fanny Anitua Zona Centro C.P. 34000 ORCID: 0000-0003-4502-4017

\section{Coautores:}

Martha Angélica Quintanar-Escorza: martha.quintanar@ ujed.mx

ORCID: 0000-0003-4256-7526

Laura Ernestina Barragán Ledesma: hábil_laura@yahoo. com.mx

ORCID: 0000-0001-5929-1648

Conflicto de intereses: los autores declaran no tener conflictos de interés.

Fuente de financiamiento: autofinanciado.

Editor:

Juan Carlos Cuevas-González

Universidad Autónoma de Ciudad Juárez, México.

Recibido: 28/10/21

Aceptado: $15 / 11 / 21$

Publicado: $21 / 01 / 22$ 


\section{Introducción}

La enfermedad por coronavirus 2019 (COVID-19) es una infección respiratoria aguda grave causada por el síndrome respiratorio agudo coronavirus 2 (SARSCoV-2) altamente transmisible, la cual ha sido declarada pandemia por la Organización Mundial de la Salud ${ }^{1}$. En concordancia con las políticas gubernamentales para la prevención sanitaria y aislamiento social, se aprobó la virtualidad de todo el sistema de educación, incluyendo carreras con un fuerte componente práctico como las de Ciencias de la Salud; lo cual conllevó a la adaptación de planes y programas de estudio, así como a la inmersión de utilización de plataformas virtuales, generando un descubrimiento de nuevas formas de aprendizaje tanto en el área teórica y práctica, sin embargo al mismo tiempo se generaron ciertas repercusiones en destrezas y habilidades de tipo prácticas y psicomotriz; abriendo además, una brecha entre la desigualdad en el acceso de las tecnologías ${ }^{2}$.

\section{Virtualidad del proceso de enseñanza aprendizaje en áreas de Ciencias de la Salud}

En la actualidad la educación virtual se volvió rápidamente una herramienta esencial para el quehacer del proceso de enseñanza- aprendizaje, en donde las plataformas de uso cotidiano y las redes sociales se volvieron básicas para el desarrollo de los contenidos académicos, ya que a través de ellas se comunicaba y se divulgaba los contenidos teóricos ${ }^{3}$.

Sin embargo, pese a haberse obtenido beneficios con la educación virtual, no sustituyen las actividades prácticas de competencia de las carreras de Ciencias de la Salud, en donde los alumnos sean capases de adquirir aprendizaje didáctico y práctica clínica, para un desarrollo de un profesional competente como es el caso de los estudiantes de las carreras de odontología, medicina, enfermería, entre otros ${ }^{4}$. En el caso de los estudiantes de las áreas de Ciencias de la Salud, se presenta un reto más fuerte debido al impacto significativo que ha tenido sobre la interrupción de la práctica clínica, afectando gravemente la confianza y preparación de los estudiantes, tal como lo demuestra un estudio realizado en Reino Unido a los estudiantes de medicina de último año además de su repercusión en la salud ${ }^{5}$.

\section{Efectos de la educación virtual en la salud en estudiantes de las áreas de Ciencias de la Salud}

Las exigencias del mundo académico de las áreas de ciencias de la salud las cuales han sido vinculadas al estrés generado por la tensión física y emocional por la cual pasan los estudiantes. Así mismo han sido estudiados aspectos como el rendimiento académico, el auto concepto académico, en las creencias de autoeficacia y la salud ${ }^{6}$, sin embargo, la mayoría de las investigaciones han sido realizadas en condiciones diferentes a la situación pandémica actual, y que abordan el estrés de los estudiantes y en menor medida el de los docentes.
Los desórdenes de ansiedad presentados por estudiantes de las carreras de ciencias de la salud han sido conceptualizados como una respuesta cognoscitiva, comportamental y fisiológica, la cual puede ser excesiva o prolongada, ante una situación percibida como amenazante, e incluso se presenta por algo que es reconocido por una idea sobre eventos que aún no ocurren, provocando en el sujeto una respuesta tanto emocional como psicofísica 7. La ansiedad puede presentarse en tres formas: normal, patológica y generalizada. Sin embargo, existe un nivel de ansiedad útil, funcional o necesaria que moviliza a actuar. Por otro lado, cuando esta supera ciertos límites ocasiona consecuencias desadaptativas ${ }^{8,9}$. Se han identificado dos tipos de ansiedad, la de estado y la de rasgo de acuerdo a Spielberg ${ }^{10}$, la cual de acuerdo al estado define la intensidad de las sensaciones de tensión, aprehensión, nerviosismo y preocupación que presenta un individuo en un momento en particular. En la de rasgo, se describen las diferencias que se presentan individualmente que permanecen relativamente estables a través del tiempo. Al respecto se ha descrito en la literatura hallazgos de estudios psicológicos posteriores a estudiantes sometidos a cuarentena, en donde los resultados psicológicos durante la cuarentena indican que el 7\% mostraba síntomas de ansiedad y el $17 \%$ mostraba sentimientos de ira, mientras que 4-6 meses después de la cuarentena estos síntomas se habían reducido al 3\% y al 6\% respectivamente ${ }^{11}$. Así mismo se ha establecido que los estados psíquicos (frustración y ansiedad) y los hábitos parafuncionales pueden ocasionar desórdenes funcionales del sistema estomatognático, específicamente en la articulación temporomandibular (ATM). La ATM es una de las articulaciones más complejas, por su estrecha relación con los órganos dentarios en oclusión y la participación sincronizada de las articulaciones asía ambos lados. Cuando la ATM no funciona correctamente se menciona que hay una disfunción temporomandibular, las cuales forman un grupo de manifestaciones clínicas caracterizadas por dolor de los músculos de la masticación, ATM, tejidos duros y blandos de la cavidad bucal; limitación de función y ruidos articulares, que son de origen multifactorial y en los que intervienen estados parafuncionales y factores psíquicos, como ansiedad, que a la vez ocasiona tensión, dolor y compromiso de la función ${ }^{12,13}$. Dentro de los signos y síntomas relacionados con los problemas de DTM se incluye: síntomas reportados como el dolor en la apertura y cierre de la boca, otalgia, cefaleas continuas y durante la función masticatoria y vértigo; mientras que los signos clínicos constituyen ruidos, brincos, limitaciones en apertura bucal, desplazamientos mandibulares en función, sensibilidad muscular, que afectan la calidad de vida de los sujetos ${ }^{14}$.

En un estudio realizado por Alavedra Martinez y cols. en el año 2021, se observó la presencia de síndrome de burnout en un rango del 8 al 56\% en estudiantes que cursaban una carrera universitaria entre los años $2010 \mathrm{al}$ 2015, por lo que se prevé que esta prevalencia aumente en los próximos ańos debido a la pandemia, en donde los estudiantes no solo deben de preocuparse por la adquisición y desarrollo de prácticas clínicas durante y 
después de terminar la carrera, sino sumándole una serie de factores externos generados por la situación como la sobre carga física y emocional, la pérdida de un familiar por la infección del virus, la economía familiar e incluso la desigualdad de acceso a la conexión a internet para tomar las clases ${ }^{15}$.

\section{Status quo de la educación en el área de Ciencias de la Salud}

Si bien se ha logrado evitar el rezago educativo a través de la implementación de herramientas de enseñanza aprendizaje que abarcan desde la utilización de métodos didácticos como el uso de simuladores modernos, programas de realidad virtual compatibles para con computadora, entre otros; el adiestramiento y la adquisición de destreza y habilidades que proporciona el campo preclínico y clínico no es equiparable a la utilización de estas herramientas innovadoras ${ }^{16}$, por lo que no deben ser considerados como suficientes para sustituir aprendizaje didáctico y práctica que otorga el campo clínico, sino más bien como una ventana de oportunidad para cultivar atributos personales que permitan hacer frente a la adversidad a un profesional en el área de la salud ${ }^{17}$. Sin embargo, serán necesarias futuras investigaciones para medir el impacto real en la efectividad de la implementación de la educación virtual en Ciencias de la Salud para mantener su calidad.

\section{Conclusión}

Las metodologías de enseñanza-aprendizaje en las carreras de Ciencias de la Salud adoptadas tras la situación pandémica mundial del SARS-CoV-2, pueden contribuir a cambiar positivamente el panorama futuro de la educación en estas áreas con un enfoque holístico sin precedente; en el cual se reinvente la educación en Ciencias de la Salud con el descubrimiento de herramientas de aprendizaje en el área teórica y el desarrollo de habilidades prácticas y destreza psicomotriz desde un punto de vista innovador, humanista e integrador.

\section{Referencias bibliográficas}

1. World Health Organization (WHO). WHO Director-General's Opening Remarks At The Media Briefing On COVID-19. 2020. [Consultado el 09 de noviembre 2021]. Disponible en: https://www.who.int/ director-general/speeches/detail/who-director-generals-opening-remarks-at-the-media-briefing-on-covid-19--. 11-march-2020

2. Ruiz-Aquino, M. El desafío de la presencialidad a la virtualidad en la educación superior en tiempos de pandemia. Desafios, 2020;11(1):e136-e136.

3. Mamani Aracayo YK, Quispe Venegas AP, Luna Mazzola I. ¿Es compatible la virtualidad con la educación odontológica? Una perspectiva estudiantil. Odontol Sanmarquina [Internet]. 5 de octubre de 2021 [consultado el 9 de noviembre 2021]; 24(4):399-400. Disponible en: https://revistasinvestigacion.unmsm.edu.pe/index.php/ odont/article/view/21081.
4. Sukumar S, Dracopoulos SA, Martin FE. Dental education in the time of SARS-CoV-2. European Journal of Dental Education, 2021;25(2):325-331.

5. Choi B, Jegatheeswaran L, Minocha A, Alhilani M, Nakhoul M, Mutengesa E. The impact of the COVID-19 pandemic on final year medical students in the United Kingdom: a national survey. BMC Med Educ. 2020 Jun 29;20(1):206. DOI: 10.1186/s12909-020-02117-1.

6. González Velázquez L. Estrés académico en estudiantes universitarios asociado a la pandemia por COVID-19. Espacio I+ D: Innovación más Desarrollo. 2020;9(25). DOI: 10.31644/IMASD.25.2020.a10

7. Martínez-Otero V. Ansiedad en estudiantes universitarios: estudio de una muestra de alumnos de la Facultad de Educación. Ensayos. 2014 [consultado el 15 mayo 2018]; 29(2):63-78. Disponible en: https://revista.uclm. es/index.php/ensayos/article/view/384/562.

8. Mayo Pais ME, González Freire B, Moreira Trillo V. Ansiedad ante los exámenes en la universidad: estudio de caso único. Rev Ansiedad Estrés. 2015;21(1):21-33.

9. Cardona Arias JA, Pérez Restrepo D, Rivera Ocampo S, Gómez Martínez J, Reyes A. Prevalencia de ansiedad en estudiantes universitarios. Rev Diversitas. 2015[consultado el 15 mayo 2018]; 11(1):79-89. Disponible en: https://www.redalyc.org/pdf/679/67943296005.pdf

10. Spielberger CD, Gorsuch RL, Lushene RE. Manual de cuestionario de ansiedad Estado-Rasgo. 9na ed. Madrid: Tea Ediciones S.A.U; 2015 [consultado el 15 de diciembre 2018]. Disponible en: http://www.web.teaediciones. com/Ejemplos/STAI-Manual-Extracto.pdf

11. Brooks SK, Webster RK, Smith LE, Woodland L, Wessely S, Greenberg N, et al. Lancet. 2020;395:912-20. DOI: 10.1016/ S0140-6736(20)30460-8.

12. Rojas Martínez C, Lozano Castro FE. Diagnóstico clínico y aspecto psicosocial de trastornos temporomandibulares según el índice CDI/TTM en estudiantes de odontología. Rev Estomatol Herediana. 2014[consultado el 15 de mayo 2018]; 24(4):229-238. Disponible en: http://www.scielo.org.pe/pdf/reh/v24n4/a04v24n4.pdf;

13. García LA, Lehmann JM, Loeza D. Apertura máxima mandibular asociada a grado de disfunción temporomandibular en pacientes de una clínica de postgrado en ortodoncia. Rev ADM. 2018[consultado el 15 de mayo 2018]; 75(1):26-33. Disponible en: https://www.medigraphic.com/pdfs/adm/od-2018/od181d.pdf).

14. Domínguez Torres L, Guerra Pérez PE, Castañeda Molerio R. La disfunción temporomandibular y su relación con la ansiedad y los hábitos parafuncionales. Mediciego. 2013 [consultado el 15/ de mayo 2018]; 19(2):3-12. Disponible en: https://www.medigraphic.com/pdfs/mediciego/mdc-2013/mdc132b.pdf.

15. Alavedra-Martínez JM, Cárdenas-Luján WJ. Burnout académico odontológico durante la pandemia por COVID -19. Odontol Sanmarquina [Internet]. 1 de julio de 2021 [consultado el 9 de noviembre 2021]; 24(3):3134. Disponible en: https://revistasinvestigacion.unmsm. edu.pe/index.php/odont/article/view/19920.

16. Wilcha RJ. Effectiveness of Virtual Medical Teaching During the COVID-19 Crisis: Systematic Review. JMIR Med Educ. 2020;6(2):e20963. DOI: 10.2196/20963. 
17. Sani I, Hamza Y, Chedid Y, Amalendran J, Hamza N. Understanding the consequence of COVID-19 on undergraduate medical education: Medical students' perspective. Ann Med Surg (Lond). 2020;5;58:117-119. DOI: 10.1016/j.amsu.2020.08.045. 